alteration accrual in adenoma progression. A cost-effective test to more accurately define the cohort of patients that will never progress to CRC would reduce the burden of procedures on both the patient and NHS.

Methods FFPE adenoma tissue resected from patients who subsequently developed CRC (progressors) and matched adenomas from patients who remained cancer-free for $5+$ years from the date of polypectomy (non-progressors) from a single-centre hospital archive (2008-2014) were analysed using low pass whole genome sequencing (LP-WGS). All adenomas were sequenced to a depth of $>0.1 \mathrm{x}$ on an Illumina platform and CNA burden was investigated.

Results In this case-control study, progressors $n=12$ have a greater CNA burden than non-progressors $n=37$, with $>0.05 \%$ of the genome altered in progressors and $<0.01 \%$ in non-progressors, $p=0.292$. The number of distinct copy-number segments were analysed to compare the presence of candidate CNAs. Gains were seen in chromosomes 7, 9 and 12 $(>25 \%)$ and losses in $18(>10 \%)$ in the progressor cohort. In comparison, minimal chromosomal changes were seen in nonprogressors.

Conclusions Adenomas from people who subsequent progressor to cancer may have a greater percentage of the genome altered when compared to non-progressors, with the majority of non-progressor adenomas having little or no genomic alterations. Larger sample sizes are required to confirm this. In the future, it is conceivable that patients with high burden of genomic alterations in their adenomas would be offered more intensive follow-up surveillance that low-burden adenoma patients.

\section{EVALUATING ORAL AND INTRAVENOUS IRON THERAPY ON BACTERIAL POPULATIONS IN NORMAL MUCOSA AND COLORECTAL TUMOUR}

${ }^{1}$ Oliver Phipps*, ${ }^{2}$ Mohammed N Quraishi, ${ }^{1,3}$ Aditi Kumar, ${ }^{4}$ Edward A Dickson, ${ }^{4}$ Oliver Ng, ${ }^{4}$ Austin G Acheson, ${ }^{2}$ Andrew D Beggs, ${ }^{1}$ Hafid $O$ Al-Hassi, ${ }^{1,3}$ Matthew J Brookes. 'University of Wolverhampton, UK; ${ }^{2}$ Institute of Cancer and Genomic Sciences, University of Birmingham, UK; ${ }^{3}$ New Cross Hospital, Wolverhampton, UK; ${ }^{4}$ The National Institute for Health Research, University of Nottingham, UK

\subsection{6/gutjnl-2020-bsgcampus.54}

Introduction Anaemia is prevalent in around 60\% of colorectal cancer (CRC) patients, mostly due to iron deficiency anaemia (IDA) and typically treated with oral iron supplements. However, this may increase the availability of luminal iron to gut bacteria leading to bacterial growth. This may potentially promote microbial dysbiosis, favouring the growth of pathogenic bacteria at the expense of commensal bacteria. Many pathogenic bacteria have heightened iron acquisition mechanism which aid their virulence, which can contribute to tumour promoting inflammation. To assess this, we compared bacterial populations and systemic cytokine production in CRC patients with IDA treated with oral or intravenous iron supplements.

Methods Patients with CRC and IDA received oral-ferrous sulphate (OI) $(n=20)$ or intravenous ferric carboxymaltose (IVI) $(n=20)$. Normal and tumour tissues were obtained post-surgery and analysed for mucosal adherent gut microbiota using $16 \mathrm{~S}$ rRNA profiling. Bacterial richness was assessed using the Chao1 test and $\alpha$-diversity was assessed using the Phylogenetic and Shannon index tests. Systemic cytokine levels were measured in the serum before and after treatment using a cytokine multiplex assay.

Results Species richness was significantly higher in normal mucosa from the OI treatment group compared to the IVI group $(p=0.033)$. Likewise, species $\alpha$-diversity in normal mucosa was significantly greater in OI treated patients (Phylogenetic $p=0.037$, Shannon $p=0.036$ ). However, tumours showed no differences in species richness or $\alpha$-diversity between treatment groups. Following OI treatment, serum levels of the pro-inflammatory cytokines IL-1b and IL-12p40 were significantly increased $(p=0.01$ and $p=0.03)$, respectively, and the anti-inflammatory cytokine IL-4 levels were significantly reduced $(p=0.01)$. In contrast, no changes in these cytokines were observed in the IVI group.

Conclusion OI therapy increased bacterial richness and $\alpha$ diversity in normal colonic mucosa and contributed to systemic inflammation in CRC patients. However, the tumour microbiota seems to be protected against increased gut iron, with no difference between OI and IVI therapy. This may be due to pre-existing dysbiosis within the cancer; hence, iron influence may be restricted in the tumours. Ongoing work will assess the abundance and diversity of protective and pathogenic bacteria to determine if these are causative in the systemic inflammation observed with OI therapy.

\section{LACTULOSE INCREASES SMALL BOWEL BUT NOT COLONIC WATER CONTENT; QUESTIONING THE OSMOTIC LAXATIVE DOGMA}

${ }^{1,2}$ David Gunn*, ${ }^{1,2}$ Caroline Yeldho, ${ }^{1,3}$ Caroline Hoad, ${ }^{1,2}$ Luca Marciani, ${ }^{1,2}$ Robin Spiller ${ }^{1}$ NIHR Nottingham Biomedical Research Centre, Nottingham, UK; ${ }^{2}$ Nottingham Digestive Diseases Centre, University of Nottingham, Nottingham, UK; ${ }^{3}$ Sir Peter Mansfield Imaging Centre, University of Nottingham, Nottingham, UK

\subsection{6/gutjnl-2020-bsgcampus.55}

Introduction Lactulose is widely perceived as an osmotic laxative which increases small bowel water content but its impact on large bowel water content is unclear. Ondansetron has been shown to slow left sided colonic transit but whether this is due to reduced secretions, enhanced absorption or solely altered motility is unknown. Our aim was to test the effect of therapeutic doses of lactulose and whether ondansetron altered its laxative effect.

Methods This was a double-blind, randomised, two-treatment crossover study in 16 healthy volunteers who attended for 2 study periods. Participants received the study drug (either 8 mg ondansetron or placebo) and had MRI scans fasted then every 2 hours for 6 hours after a rice pudding meal. They then received lactulose $(20 \mathrm{ml}[39 \mathrm{mmol}])$ twice daily and the study drug three times daily for 36 hours. On day 3 they had lactulose and the study drug, and further MRI scans every 2 hours for 4 hours. Measurements included small bowel water content (SBWC), magnetic resonance T1 relaxometry to assess water content in the ascending colon (AC) (T1 has previously shown to be directly proportion to stool $\%$ water) and gut transit from the weighted average position of transit markers ingested on Day 2.

Results In the placebo arm, lactulose increased small bowel water content maximally at 120 minutes (figure 1), with an increase of $89 \mathrm{ml}(95 \% \mathrm{CI} 32 \mathrm{ml}$ to $145 \mathrm{ml})$ compared to the test meal without lactulose. Lactulose significantly increased AUC SBWC from 0-240 minutes (43.3 [25.0] 1 . 